CONTINUAT!ON APPLICATION FOR DE-FG03-88ER45375

DOE/ER/45375- -T'1

DE92 012600

\title{
SEGMENTAL INTERPENETRATION AT POLYMER INTERFACES
}

\author{
T. P. Russell \\ W. W. Fleming \\ IBM Almaden Research Center
}

6.50) Il larry Road

San Jose, CA 9.5120

\section{DISCLAIMER}

This report was prepared as an account of work sponsored by an agency of the United States Government. Neither the United States Government nor any agency thereof, nor any of their employees, makes any warranty, express or implied, or assumes any legal liability or responsibility for the accuracy, completeness, or usefulness of any information, apparatus, product, or process disclosed, or represents that its use would not infringe privately owned rights. Reference herein to any specific commercial product, process, or service by trade name, trademark, manufacturer, or otherwise does not necessarily constitute or imply its endorsement, recommendation, or favoring by the United States Government or any agency thereof. The views and opinions of authors expressed herein do not necessarily state or reflect those of the United States Government or any agency thereof. 
PROGRESS REPORT

During the first year of this proposal significant strides have been male in understanding the nature of the interface in symmetric, diblock copolymers and the nature of the interface formed between immiscible homopolymers. Imphasis has heen placed on the symmetric, diblock copolymers of polystyrene (PS) and polymethylmethacrylate (PMMA) since these diblock copolymers and the corresponding homopolymers are available over a wide range of molecular weights with narrow molecular weight distributions. In addition, the homopolymers and copolymers can be obtained with different levels of deuteration. Thus, these materials form a model system by which systematic studies on the interfacial behavior of homopolymers and block copolymers can be performed.

The work during this first year can be broken down into live different categories where significant advances have been made. These are:

1. Interaction parameter between PS and PMMA

2. Surface induced ordering of phase mixed diblock copolymers

3. Surface induced ordering of microphase scparated diblock copolymers

4. Molecular weight dependence of the features of the morphology of diblock copolymers

5. Interface between PS and PMMA homopolymers

Each of these will be treated scparately below.

\section{INTERACTION PARAMETER BEWTEEN PS AND PMMA}

Using small angle neutron scattcring, symmetric, diblock PS/PMMA copolymers, where the total molecular weight of the copolymer was 27,660 with a polydispersity of 1.084 , the Flory-Huggins interaction parameter, $\chi$, was determined. $\Lambda t$ temperatures above the glass transition temperatures of the individual blocks the copolymer exists in the phase mixed state. The small angle neutron scattering profile is given by the well known correlation hole scattering function where the position of the maximum in the profile is dictated primarily by the chain conformation and the shape is governed predominantly by $\chi$. Experiments were 
performed at several temperatures from which the temperature dependence of $\chi$ was found to he given by $\chi=0.0284+\frac{3.9}{\mathrm{I}}$. There are several surprising features of these results. Firstly, it is shown that $\chi$ depends very weakly on temperature. Secondly, the temperature invariant portion of $\chi$, i.c. the entropic contribution to $\chi$, dominates the interaction parameter. These results are quite different from the usual behavior found in diblock copolymers.

From the value of the molecular weight, expressed as the number of segments in the copolymer chain, $\mathrm{N}$, the temperature at which the copolymer encounters a transition from an ordered state, where a lamellar microdomain morphology is expected, to a phase mixed state can be determined. The critical value of $\chi \mathrm{N}$ is 10.5. For the copolymer of studied here a critical temperature of $65^{\circ} \mathrm{C}$ is found. What is quite surprising is that by increasing $\mathrm{N}$ by only 28 results in a critical temperature in excess of $225^{\circ} \mathrm{C}$, which is quite close to the temperature at which substantial thermal degradation occurs. Consequently, the window in molecular weight over which the order-disorder transition occurs is quite small.

Full details of this study can be found in the attached article recently submitted for publication in Macromolecules ( $\Lambda$ ppendix I).

\section{SURFACE ORDERING OF PHASE MIXED COPOLYMERS}

The specular reflectivity of neutrons was used to investigate the behavior of a PS/PMMA diblock copolymer at temperatures above the order-disorder transition temperature. In the disordered state the copolymer films near the air surface and near the interface with the substate (in this case silicon) were not found to he homogencous. At the air surface the concentration of PS, the lower surface energy component, was found to be 0.65 in volume fraction which is in excess of the 0.5 volume fraction characteristic of the bulk material. $\Lambda \mathrm{s}$ one penetrates into the film the concentration of PS was found to he described by $\phi(7)=\phi_{\mathrm{s}} \exp (-7 / \xi) \cos (2 \pi \% / 1)+\bar{\phi}$ where $\phi(z)$ is the volume fraction of PS as a function of depth $\%, \phi_{\mathrm{s}}$ is the excess concentration of PS at the surface, 1 , is the fluctuation length of the copolymer, $\xi$ is a characteristic correlation length, and $\bar{\phi}$ is the average bulk concentration. The latter two parameters are matcrials constants and are found to be given by $\mathrm{L}=150 \mathrm{~A}$ and 
$\xi=97 \pm 5 \AA$. From the value of the Flory-lluggins interaction parameter and the molecular weight of the conolymer both $\mathrm{L}$, and $\xi$ can be theoretically calculated. Irom theory these are found to be $L=150 \AA$ and $\xi=104 \AA$. in excellent agreement with the experimental results.

PMM $\Lambda$ is also known to be located preferentially at the silicon/copolymer interface. From these same measurements it was found that the concentration of PMM $\mathrm{PAt}$ the Si surface was nearly unity. Again, the concentration of PMM $\mathrm{P}$ from the Si surface was found to be given by an exponentially damped cosine function whose characteristic parameters were identical to those found at the air surface.

The full details of the experiments and the results are found in the attached article published in Physical Review Letters (Appendix II). In summary, it is clear that the presence of an interface perturbs the concentration of copolymer segments such that the concentration near the surface is not uniform.

\section{SURFACE INDUCED ORDERING OF MICROPHASE SEPARATED COPOLYMERS}

It has been shown that symmetric, diblock copolymers of PS and PMMA, at temperatures below the order-disorder transition temperature, that the lamellar microdomain morphology orients parallel to the surfaces. This orientation, induced by the presence of an interface and the preferential adsorption of one component to the interface, propagates over very large distances in the specimen. In fact, for specimens with total thicknesses of $5 \times 10^{3} \mathrm{~A}$ or less this oriented lamellar morphology saturates the entire film. This orientation coupled with the high spatial resoluticn of neutron reflectivity was used to obtain detailed information on the morphology of the copolymers. In the first study of its kind, we showed that the interface between the PS and PMMA microdomains was $47 \pm 2 \AA$. The importance of this study lay in the fact that we were able to resolve the width of the interface with an accuracy unmatched by other studies up to now. This has permitted a precise comparison with theoretical predictions of the interface width. Theoretically, knowing the statistical segment length of the copolymer and the filory-lluggins interaction parameter (hoth of which were 
obtained from the above mentioned neutron scattering studies), the width of the interface is calculated as being $28 \AA$. This difference is quite real and points to shortcomings of current theoretical understanding of the interface in copolymers.

The first results of this study can be found in the attached Physical Review Letters article (Appendix II).

\section{MOLECULAR WEIGHT DEPENDENCE OF MORPHOLOGY}

In a subsequent study a series of PS/PMMA symmetric, diblock copolymers were investigated where either block was perdeuterated and where the total molecular weight of the copolymer was varied from ca. 15,000 to ca. 150,000 . As hefore, copolymers with narrow molecular weight distributions were used in all studies. Specimens were prepared by solution casting films of the copolymers onto silicon substrates followed by annealing for 72 hours above the glass transition temperature so as to induced the strong oricntation of the lamellar microdomian morphology. These specimens were then investigated by neutron reflectivity to characterize in detail the molecular weight dependence of the lamellar microdomian morphology of the diblock copolymers.

Several important resulis stemmed from these studies. Fistly, it was found that the interface between the PS and PMM $\triangle$ microdomains was $4.5 \pm 5 \mathrm{~A}$ independent of the molecular weight of the copolymer. The molecular weight invariance of the interface is consistent with theoretical predictions. However, theoretically, from the statistical segment length of the copolymer and the Flory-Huggins interaction pa...neter, the width of the interface calculated is $28 \AA$. This difference is quite real and points to a deficiency in the theoretical arguments. Sccondly, the variation in the concentration of segments across the interface is given very closely by a hyperbolic tangent function. This is the first time that the functional form of the interface could be preciscly resolved. Using techniques other than neutron reflectivity it has been impossible to define the functional form of the interface. These two results represent an important step forward in the understanding of the interfacial behavior of diblock copolymers. 
Of great importance to the successful completion of the research outlined in the initial proposal is the accurate characterization of the interface between two homopolymer phases. Such measurements have been, heretofore, most dificult with any degree of precision. To address this issue a bilayer of deuterated PS on top of normal PMMA was prepared as follows. PMM having a molecular weight of 150,00 with a narrow molecular weight distribution was solution cast onto a silicon substrate. This film was annealed at $150^{\circ} \mathrm{C}$. for 48 hours under vacuum to remove the solvent and to allow the film to relax. Onto a separate substrate a film of deuterated PS having a molecular weight of 150,00 with a narrow molecular weight distribution was cast from a toluene solution. The film was dried and then floated off onto a pool of water. Using the substrate coated with the PMMA, the deuterated PS was retrieved from the water surface, forming the desired bilayer. The bilayer specimen was then heated to $170^{\circ} \mathrm{C}$ under vacuum for a period of 96 hours to remove any remaining solvent or entrapped air, and to allow the bilayered specimen to come to equilibrium. This specimen vas then investigated by neutron reflectivity.

The neutron reflectivity profiles clearly showed that the interface between the homopolymer layers was best described by a hyperbolic tangent function with an overall interfacial width of $4.5 \pm 5 \AA$. This result is identical to that seen in the case of the copolymers which is consistent with theoretical arguments. Again, however, ? is a discrepancy between the absolute size of the interface calculated from theory and that seen experimentally. 


\section{PUBLICATIONS}

Stemming from the support of the Department of Incrgy the following publications have arisen. The state of the publication is given in each case.

1. "Neutron Reflectivity Studies of the Surface Induced Ordering of Diblock Copolymer Films", S.II.Anastasiadis, T.P.Russell, S.K.Satija and C.F.Majkrzak, Phys. Rev. Lett., 62, 1852(1989).

2. "Temperature Dependence of the Interaction Parameter of Polystyrene and Polymethylmethacrylate", T.P.Russell, R.P.Iljelm, Ir., and P.A.Secger, Macromolecules, submitted.

3. "Block Copolymer Behavior in Thin Films", T.P.Russell, V.R.Deline, V.S.Wakharkar and G.Coulon, Mat. Res. Soc. Bull, accepted.

4. "The Morphology of Symmetric Diblock Copolymers as Revealed by Neutron Reflectivity", S.II.Anastasiadis, T.P.Russcll, S.K.Satija and C.I.Majkrzak, J.Chem.Phys., in preparation. 
PLANS FOR 1989-1990

It is clcar that a substantial understanding of the interfacial behavior of diblock copolymers and of the interfacial behavior of homopolymers has heen obtained. This has becn the first step in accomplishing the rescarch goals stated in the original. It is also apparent that we have cmphasized the use of neutron reflectivity as a means of quantitatively examining the morphology in thin films of the copolymers. This deviates from the original proposal, since we had initially proposed the combined use of small angle $x$-ray and neutron scattcring with cross polarization magic angle spinning nuclear magnetic resonance. This deviation was mandated by the advent of the neutron reflectivity technique and our being in a key position to take advantage of this technique in a timely manner. This deviation has provided us with an exceptional means by which we can accomplish the goals of the proposed rescarch. This does not mean that we not pursuc the magnetic resonance approach. However, quantitative utilization of the technique, as stated in the proposal, requires a significant amount of calibration which are underway.

In the upcoming year we plan to continue the studies on the copolymer and the homopolymer/copolymer mixtures by both the neutron reflectivity technique and the combined scattering/magnetic resonance techniques. In this way we will be best assured of completing the aim of the proposed research which is to understand the segmental interpenetration at polymer-polymer interfaces. In particular we list below under separate headings the subjects that will be pursued in the upcoming year.

\section{FILM THICKNESS EFFECTS ON COPOLYMER MORPHOLOGY}

In order to 'snderstand the forces that are active in the formation of a copolymer morphology and to understand the behavior of copolymers in confined spaces, as for example at an interface, it is of interest and importance to investigate the efrect of rilm thickness on the copolymer morphology. In these studies we plan to investigate copolymer films where the total thickness of the film is comparahle to and less than a single lamellar period. These studies will be aimed at understanding when and how the microphase separated morphology is 
perturbed by the severe houndary conditions place on the copolymer by the presence of the substrate and air interfaces.

\section{TEMPERATURE DEPENDENCE OF SURFACE INDUCED GRADIENTS}

From our studies on the behavior of diblock copolymers in the phase mixed state, it was clear that the presence of interface dramatically perturbs the concentration of copolymer segments near the interface. In fact, our results showed remarkahle agreement with theoretical predictions. To understand the interfacial behavior further it is of importance to understand the dependence of the surface induced perturbations on temperature. 'Theoretically, it would be predicted that the extent to which the surface effects propagate into the bulk of the copolymer will depend upon $\left((\chi N)_{\mathrm{s}}-(\chi N)\right)^{0.5}$ where $(\chi N)_{\mathrm{s}}$ is 10.5 for a symmetric, diblock copolymer. This effectively means that the correlation length will increase dramatically as the order-disorder transition temperature is approached. Quantitatively confirmation of this prediction is important for the use of copolymeric matcrials as adhesion promoters and surfactants.

\section{HOMOPOLYMER/COPOLYMER MIXTURES}

Directly related to the question of the segmental interpenetration of segments at interfaces is the segment density profiles in specimens where the concentration of the copolymer is high and that of the homopolymer is low. In such a case, we have shown that lige orientation of the copolymer microdomains parallel to the surface is retained in thin films. Using neutron reflectivity and the appropriate combinations of deuterated or protonated homopolymers with labelled copolymers, it will be possible to evaluate the segment density distribution of the homopolymers and copolymers in the films. This information will yicld directly the extent of segmental overlap hetween the homopolymer and the copolymer and will yield the extent of penetration of the homopolymers across the interface dominated by the copolymer. 


\section{COPOLYMERS AT INTERFACES}

We have already performed a series of secondary ion mass spectroscopy studies where we are attempting to characterize the segregation of copolymers to the interface hetween two homopolymers. Several different approaches have been taken. In the first case bilayer films of mixtures of PS with PS/PMMA and of PMMA with PS/PMMA have been prepared, Here, the segregation of the copolymer to the interface hetween the homopolymers is clearly seen. In the second case, a trilayer specimen is prepared comprised of a layer of PS, a thin copolymer layer and a layer of PMM concentration and temperature dependence of these different preparations using secondary ion mass spectroscopy. Once a sufficient understanding of the parameters that control the segregation behavior of the copolymers has been obtained, neutron reflectivity will be used to probe quantitatively the segment density profiles across the interface of the two homopolymer layers. This study, however, can only be accomplished when we have gained sufficient understanding of the segregation process by the more readily accessible technique of secondary ion mass spectroscopy. 


\section{CONTINUANCE REQUEST}

In order to perform the proposed rescarch in the upcoming year we are recuesting the continuance of proposal DE-F(303-88I:R45375. In the past year we have adhered to the overall objectives of the original proposal by studying, in detail, the interfacial behavior of pure homopolymers and thin film behavior of diblock copolymers. Having attained this knowledge we are in an advantageous position to obtain a quantitative understanding of the segmental interpenetration at polymer interfaces. Attached is a modilied budget where we have, at the request of the Department of the Inergy, Offices of Basic Inergy Sciences, reduced the requested amount by $10 \%$. $\Lambda$ s can be secn, this reduction does not mean that the amount of research performed by the principal investigators will change. The difference in the initial budget and the modified budget translates into a larger portion of the budget being assumed by the International Business Machines Corporation. It is evident that the results from the past year and the proposed plans for the upcoming year can not permit a reduction in the amount of efrort in this project. 


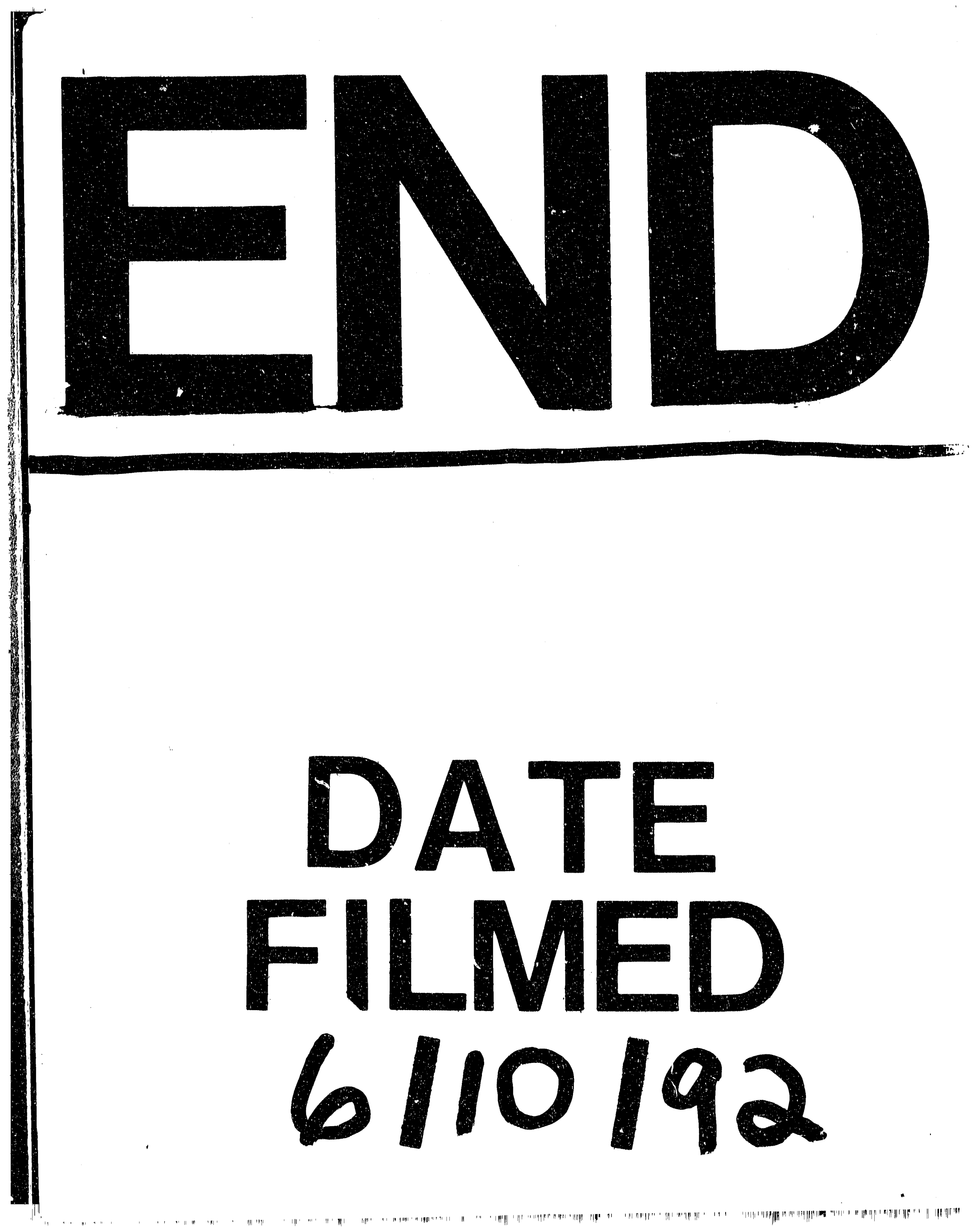

